\section{Creating a paradigm shift in plant breeding and plant phenotyping}

Innovative plant breeding strategies accessing the beneficial relationships between soil microbes and varieties that help develop to climate change and soil conditions. Dr Omirou and Dr Fasoula at the Agricultura Research Institute, Cyprus, describe how moving away from conventional multiplant, densely-grown field plots, and using innovative selection designs fitted to wididual plants grown at ulraseveral barriers to effective plant phenotyping. Their experimental planting pattern called Honeycomb Selection Designs, when combined with prognostic breeding, can evaluate plant productivity and trait performance stability in the same generation, and can plant-microbiome associations.

Plant - Microb interactions in rhizosphere

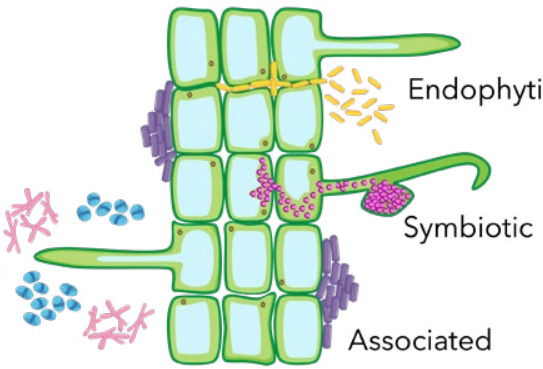

lant breeding relies on the evaluation of field performance
followed by the selection of (osirable plant phenotypes (observab The same plact gentype will produce different phenotypes, depending on the environment in which it grows. Since a plant can't move, a significa part of its environment is the specific soil spot where it grows, which may This soil heterogeneity involves various factors, including the distribution of the soil microbiome. Plants acquire many desirable tratts through their interaction with soil microbial communities, an effect which could be put to beneficial use.

Now, a new trend in plant breeding is being directed at harnessing the soil microbiome - the community of bacteria, archaea, viruses, fun and protozoa present in the soil improved abilities to benefit from associated microbiome. Soil microbial assemblages can, for example, suppress plant pathogens or enhance nutrient uptake and response to stresses like drought and salinity. By improving the interaction between plants and soil microbiomes agricultural scientists could mitigate and even reverse negative effects of climate change and of soil degradation on crop yields. The soil microbes associated with plants can be endophytic (living within a plant) or rhizospheric (present around the plant root system). Examples of differ from soil only a few metres away. the interaction of their roots with the bacteria supporting nitrogen fixation in legumes and arbuscular mycorrhiza fungi (AMF) enhancing drought nitronce. Breeding for enhanced example, could help reduce the costly application of nitrogenous fertilizers. The innovative plant breeding strategies adopted by DrOmirou and Dr Fasoula seek to exploit the inherent create more resilient varieties.

\section{ADVANTAGES OF HONEYCOMB} SELECTION DESIGNS AND

\section{PROGNOSTIC BREEDING}

pelant breeding programmes, traits depection of desired crop appropends on determining an appropriate unit of plant phenotyping breeding uses multi-plant, den grown field plots as the units of plant prectice can limit accurate phenotyping systems. It also cannot take the best advantage of current advances in genomic information. To address some methods, Dr Omirou and Dr Fasoula use innovative selection designs where individual plants are grown at ultra-wide distances to fully express their genetic potential and maximise phenotypic

in and differentiation. place indvivdua plection Designs place individual plants in triangular distances wide enough to exclude the found in traditional selection plots. properties of microbiome complexity to breeding uses multi-plant, densely of plants and their associated root of the problems with conventional grid patterns (hence 'honeycomb') at

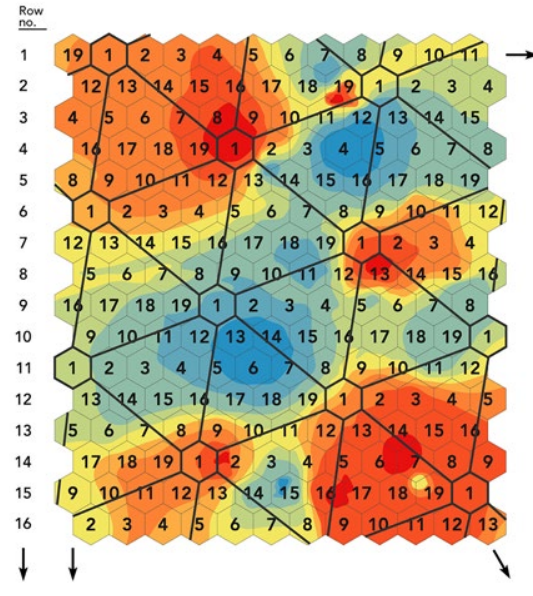

Figure 1. The replicated-19 Honeycomb Honeycomb Selection Designs and evaluates
Hons plants of 19 lines or genotypes. The numbers
represent the position of plants in the field and represent the position of plants in the field and
the hexagons represent the area exploited by each plant. The plant-to-plant distance is $1 \mathrm{~m}$
las seen in actual field photo, overleaf). The design is depicted on top of a multicolo heterogeneity of the whole field. Each colo microbial genera. Plants of each line are arranged in an ascending numerical order an are placed in the corners of moving equilater
triangular grids, exemplified here for line 1 . The grids spread across the field and sample grids ensure reliable measurement. of the trait stability of each line through the use of the thability (or homeostasis) index, $\mathrm{SI}=(\tilde{\mathrm{X}} / \mathbf{s})$ ? deviation of the plants allocated across the triangular grid of each line.

Competition for resources in denselygrown plots prevents individual pla fom maximising their phenotypic expression and realising their full genetic poten
This has not previously been

Plants acquire many desirable traits through their interaction with soil previously been for and explains why breeding for increased yield has progressed rather slowly. Competition can create large variations in plant yields and other traits of interest and introduce bias, since a breeder will not know if this is true genotypic superiority due to some plants gaining advantages at the expense of others nearby.

The Honeycomb Selection Designs overcome the effects of spatial variation in soil characteristics by the formatio of moving, complete replicates and .$$
\text { microorganisms living in the soil. The }
$$

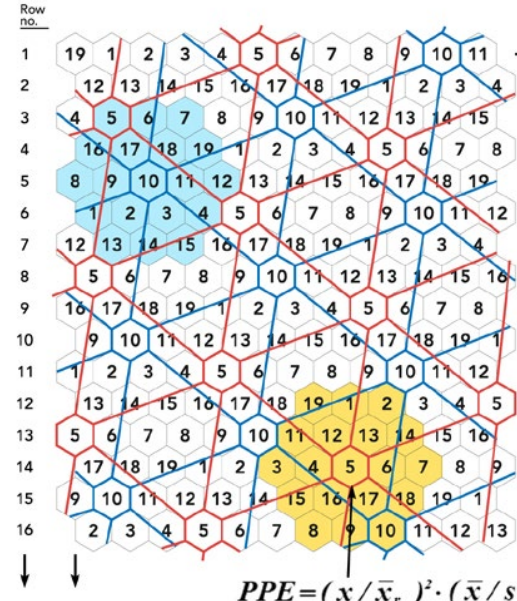

Figure 2. Additional properties of the highlighted. The same properties apply to
all Honeycomb Selection Designs. In every field position, each plant occupies the as shown for two random plants of lines no.
10 and no. 5 . Moving replicates (or moving rings) are illustrated with blue and orange shades and include representative plants of
all 19 lines. The use of the plant yield index spatial heterogeneity on single-plant yields
because $\boldsymbol{x}$ is the yield of each plant and $\bar{x}_{i}$ is the mean yield of the surrounding movingnumber unique for each plant and is the value
of its phenotyping equation PPE under the of its phenotyping equaticitions.
specific conditions

Soil heterogeneity includes a variety of factors, like availability of plant nutrients, soil structure and $\mathrm{pH}$, ability obial communities, an effect which could be put to beneficial use.

designs enable effective sampling
of the full spectrum of soil and
environmental heterogeneity, ensuring
that all plants and sibling lines are
allocated under comparable growing
conditions in both fertile and less
fertile spots. The triangular grid shown
in Figure 1 illustrates how the plants
of line no. 1 are allocated effectively
across different soil conditions and
spatial variation.
Conventional plant breeding systems
plants in the field
survive. Selection stability (or homeostasis) index secu good performance under high planting censities, as is the situation when the and all plants survive.

In a recent paper, Fasoula and colleagues outlined how these alternative approaches help to overcome the various barriers in plant phenotyping. As discussed, barriers effects of interplant competition spatial heterogeneity. Also, the limited 


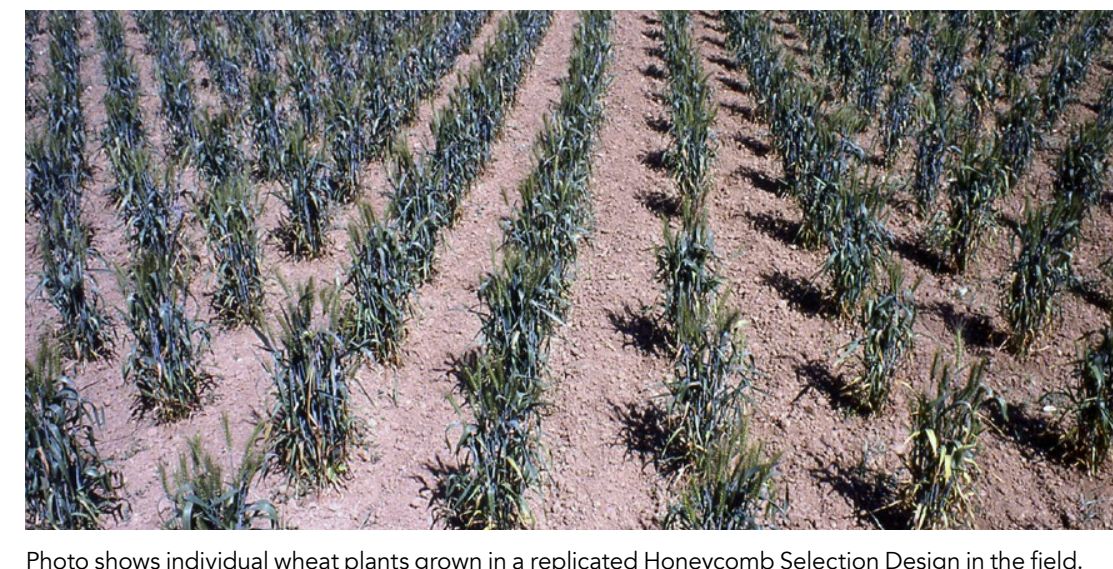

seed supplies in early segregating is the greatest limiting factor on yield issue in Honeycomb Selection Designs
with climate change. This crop is sester since they evaluate single plants. This Plant Genetic Resources and Crop Wild Relatives for which seed supplies methodology avoids the restrictions of current statistical approaches.

These Honeycomb Selection Designs can be used in all plants amenable to breeding, regardless of their mode of propagation and annual or perennial habit. The designs facilitate the possibility of mechanising and automating selection for yield and known to be associated with arbuscu mycorrhizal fungi (AMF), which have tolerance of the host chickpea plant atering the host hormone blant by enhancing the movement of water from roots to shoots.

Omirou, Fasoula and collaborators limitation on plant growth and the AMF biome in chickpea genotypes previously selected in the field using the Honeycomb Selection Design.
To further investigate specific

Honeycomb Selection Designs and the phenotyping equations of prognostic breeding can be used in all plants and categories of crops.

other important plant traits. Finally, and of particular interest here, their growth and permits selection of plants with improved root architecture, efficient in capturing resources by roots and exploiting microorganisms that can promote plant growth. This practice can facilitate ongoing global efforts for overcoming the challenges of root phenotyping in the field. The methodology is also applicable to Organic Agriculture.

\section{THE EXAMPLE OF CHICKPEA}

Chickpea is one of the most impor

legumes and is grown in and and

details, they chose two genotypes for controlled pot trials. Their recent report describes the response of the two chickpea genotypes grow under well-watered and limited wate conditions to see if water limitation affected nutrient accumulation and mycorrhizal colonisation. The responses of the AMF assemblages wociated with the plant root syste were also evaluated.

One of the chickpea genotypes showed no difference in growth or AMF colonisation when water was drought stress reduced growth drought stress reduced growth and this
AMF colonisation. The results suggest that chickpea genotypes can differ in of chickea could therefore be bred for improved drought tolerance and enhanced AMF association. The above selection methods along with availability of beneficial microbiomes wi successfully support such an endeavour

DEVELOPING NATIVE MICROBIAL SOIL GENETIC RESOURCES Selecting for plant phenotypes that attract microorganisms able to promote plant growth could provide an effective strategy for tackling the adverse conditions of climate change. dentifying the core microbiome of plant species suitable for breeding, solating the microbes and applying creete field condition could also help perferid condilions hat favour plant from relevant agricultural ecosystems will ensure the closest possible relationship between effective microbia guilds and crop plants.

Metagenomics (the study of genetic material recovered directly from environmental samples) is expected to provide advanced microbial solutions for future more sustainable agricultural practices. The researchers group leads a state-of-the-art environmental microbiology and biotechnology unit (https://magnet. moa.gov.cy/for studying the native microbial diversity of Cyprus. Base icuentories of the microbiomes are also in soil biodiversity orer time in the m agricultural areas of the country.

In addition, Dr Omirou and Dr Fasoula are working to isolate and characterise soil microbes that promote plant growth under drought, through an international project funded by the EU (https://sites.unica.it/ supreme/). The aim is to combat soil impoverishment and to reduce the use of water, fertilizers and pesticides by harnessing the microbiome potentia to stabilise soil and promote plant growth under adverse conditions. The hope is that this will eventually benefit living in semi-arid aral communities llving in semi-arid and

\section{Behind the Research}

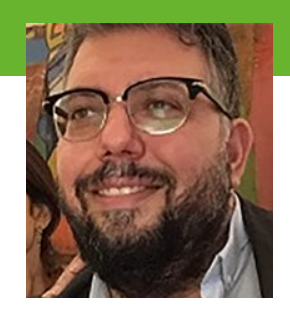

Dr Michalis

Omirou

E: michalis.omirou@ari.gov.cy
T: +35722403146

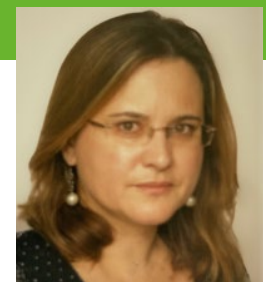

Dr Dionysia A.

Fasoula

E: dfasoula@ari.gov.cy

W: www.ari.gov.cy

Research Objectives

Drs Omirou and Fasoula are creating a national infrastructure of soil genetic resources and developing more resilient,

\section{Detail}

Dr Michalis Omirou Agricultural Research Institute PO Box: 22016, 1516 Nicosia, Cyprus

Department of Agrobiotechnology
in the Agricultural Research Institute, Nicosia, Cyprus

Bio

Dichalis Omirou leads a National metagenomics exploitation of Soil

Genetic Resources and is the Group Leader of Agricultural Microbiology.

Dr Dionysia A. Fasoula is a Plant Dreder and Molecular Geneticist, Agricultural Research Institute, Nicosia, Cyprus.

\section{References}

-Fasoula, D.A., loannides, I.M. \& Omirou, M. (2020) Phenotyping and plant breeding: overcoming the barriers.

-Kavadia, A., Omivu, M., Fasoula, D., Trajanoski, S., Andreou, E. \& loannides, I.M. (2020). Genotype and soil water availability growth stages. Applied Soil Ecology 150, 103443.

- Omirou M., loannides M.I., Fasoula D. (2019). Optimizing Resource Allocation in a Cowpea (Vigna unguiculata L. Walp.) Landrace Through Whole-Plant Field Phenotyping and Nonstop Selection to Sustain Increased Genetic Gain Across Decade. Frontiers in Plant Science 10, 949

-Fasoula, V.A. (2013). Prognostic breeding: a new paradigm for crop improvement. In Plant Breeding Reviews, Vol. 37. 297-347 (John Wiley \& Sons).

- Fasoula, D.A. \& Fasoula, V.A. (1997). Competitive ability and plant breeding. In Plant Breeding Reviews, Vol. 14. 89-138 (John Wiley \& Sons)

Fasoulas, A.C. \& Fasoula, V.A. (1995). Honeycomb selection Reviews, Vol. 13. 87-139 (John Wiley \& Sons).

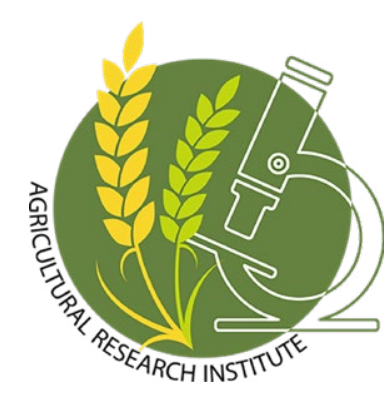

Funding PRE ERANEDO36 Foung

\section{Collaborators}

- Dr loannis M. loannides - Dr George Spyrou - Dr George Tsouloupas

\section{Personal Response}

\section{In your opinion, what is the scale of soil and} mo phent-riobiome associations?

II Soil is the cornerstone of food production inputs and climate change and of course the long term monocultures and monocropping tha are still dominating farming practices globally. The rate of soil degradation in arid and semarid regions is very high and threatens the

The challenge for scientists and policy makers is to adopt novel concepts that are able to sustain and improve soil productivity. The development of microbial tools, along with the development of resilent, density-neutral (or density-independent) even under limited growth resources without requiring higher planting densities for optimal productivity, is an integral part of this effort and could be enormously useful for a climate- 